\title{
Combination of Dichotomizers for Maximizing the Partial Area under the ROC Curve
}

\author{
Maria Teresa Ricamato and Francesco Tortorella \\ DAEIMI - Università degli Studi di Cassino \\ via G. Di Biasio 43, 03043 Cassino, Italy \\ \{mt.ricamato, tortorella\}@unicas.it
}

\begin{abstract}
In recent years, classifier combination has been of great interest for the pattern recognition community as a method to improve classification performance. The most part of combination rules are based on maximizing the accuracy and, only recently, the Area under the ROC curve (AUC) has been proposed as an alternative measure. However, there are several applications which focus only on particular regions of the ROC curve, i.e. the most relevant for the problem. In these cases, looking on a partial section of the AUC is the most suitable approach to adopt. In this paper we propose a new algorithm able to maximize only a part of the AUC by means of a linear combination of dichotomizers. Moreover, we empirically show that algorithms that maximize the AUC do not maximize the partial AUC, i.e., the two kinds of maximization are independent.
\end{abstract}

Keywords: Classifiers combination, ROC curve, partial AUC.

\section{Introduction}

Classifier combination has received considerable attention in the last years becoming an established technique for improving classification performance. In a classifier combination system, the output information of all the individual classifiers are combined in order to improve their performance. It has been proved that a successful combination rule exploits variations between individual classifiers, using their strengths to take advantages and to decrease their weaknesses. Among the various classifier combination methods previously proposed, linear classifier combination has been used mainly for its simplicity and good comprehensibility. In particular, there are some methods designed to increase the Area under the ROC curve (AUC), a more suitable performance measure than the classification accuracy [1, specially for those applications characterized by imprecise environment or imbalanced class priors [2]. AUC reduces an entire ROC curve to a single quantitative index showing classifier performance over all the false positive rate (FPR) values. However, there are many applications that are interested only to a particular range of FPRs. For example, in a biometric authentication system used to identify people, or to verify the claimed identity of registered users when entering in a protected area, a false positive is considered 
the most serious error, since it gives unauthorized users access to the systems that expressly are trying to keep them out. Another example is given by medical screening tests, where a false positive involves more expensive and sophisticated exams in order to be sorted out. In both cases, the FPR values considered are the ones that correspond to lower values, and the partial AUC [3] is the most indicate index to use, since it allows us to focus on particular regions of the ROC space. The partial AUC (pAUC) has already been used as a performance measure in applications for screening research [4] [5], but it has been given little attention to it as a performance measure in machine learning in order to build classification systems and to evaluate learning algorithms.

Our main purpose is to introduce the partial AUC measure and use it in the particular context of classifiers combination. Specifically, we propose a new algorithm able to find the weight vector in a linear combination of $K \geq 2$ dichotomizers, such that the pAUC is maximized.

The paper is organized as follow. The next section presents the pAUC index and its main properties. The proposed algorithm is analyzed in section 3 for a combination of two dichotomizers, and in section 4 for a combination of more than two dichotomizers. The performed experiments and obtained results are shown in section 5, while section 6 concludes the paper.

\section{ROC Analysis and Partial Area under the ROC Curve}

Receiver Operating Characteristics (ROC) graphs are useful for visualizing, organizing and selecting classifiers based on their performance. Given a two-class classification model, the ROC curve describes the trade-off between the fraction of correctly classified actually-positive cases (True Positive Rate, TPR) and the fraction of wrongly classified actually-negative cases (False Positive Rate, FPR), giving a description of the performance of the decision rule at different operating points.

In some cases, it is preferable to use the Area under the ROC Curve (AUC) 6] [7, a single metric able to summarize the performance of the classifiers system:

$$
A U C=\int_{0}^{1} R O C(t) d t
$$

Remembering that some applications do not use all the range of false positive rates, it is worth to introduce another summary index that considers only those FPRs between $t_{0}$ and $t_{1}$, called partial AUC (pAUC), and defined as:

$$
p A U C=\int_{t_{0}}^{t_{1}} R O C(t) d t
$$

where the interval $\left(t_{0}, t_{1}\right)$ denotes the false positive rates of interest. Its choice depends on the particular application, and it is related to the involved cost of a false positive diagnosis. 
Moreover, the pAUC can be also defined as the probability that a classifier will rank a randomly chosen positive instance higher than a randomly chosen negative one, such that this latter belongs to the $1-t_{k}$ quantiles 1 range $\left(q_{y}^{t_{1}}, q_{y}^{t_{0}}\right)$ :

$$
p A U C=P\left\{x_{i}>y_{j}, y_{j} \in\left(q_{y}^{t_{1}}, q_{y}^{t_{0}}\right)\right\}
$$

where $x_{i}=f\left(\mathbf{p}_{i}\right)$ and $y_{j}=f\left(\mathbf{n}_{j}\right)$ are the outcomes of the dichotomizer $f$ on a positive sample $\mathbf{p}_{i}$ and a negative sample $\mathbf{n}_{j}$.

In order to evaluate the pAUC of a dichotomizer avoiding to perform a numerical integration on the ROC curve, we use the non-parametric estimator [3], which is defined as:

$$
p A U C=\frac{1}{m_{P} m_{N}} \sum_{i}^{m_{P}} \sum_{j}^{m_{N}} V_{i j}^{q_{y}^{t_{0}}, q_{y}^{t_{1}}}
$$

where $m_{P}$ and $m_{N}$ are the cardinalities of the positive and negative subsets, respectively, and

$$
V_{i j}^{q_{y}^{t_{0}}, q_{y}^{t_{1}}}=I\left\{x_{i}>y_{j}, y_{j} \in\left(q_{y}^{t_{1}}, q_{y}^{t_{0}}\right)\right\}=\left\{\begin{array}{l}
1 \quad \text { if } x_{i}>y_{j} \bigwedge y_{j} \in\left(q_{y}^{t_{1}}, q_{y}^{t_{0}}\right) \\
0.5 \text { if } x_{i}=y_{j} \bigwedge y_{j} \in\left(q_{y}^{t_{1}}, q_{y}^{t_{0}}\right) \\
0 \quad \text { if } x_{i}<y_{j} \bigwedge y_{j} \in\left(q_{y}^{t_{1}}, q_{y}^{t_{0}}\right)
\end{array}\right.
$$

Since the most part of biometric and medical applications [8] work on false positive rate close to the zero value, for the following analysis we consider $t_{0}=0$. In this case, equation 5 can be rewritten as $V_{i j}^{q_{y}^{t_{1}}}=I\left\{x_{i}>y_{j}, y_{j}>q_{y}^{t_{1}}\right\}$ 2.

\section{Linear Combination of Two Dichotomizers}

Let us consider a set $T$ of samples, and define the outputs of two generic dichotomizers $f_{h}$ and $f_{k}$ on two positive and negative samples $\mathbf{p}_{i}$ and $\mathbf{n}_{j}$ :

$$
x_{i}^{h}=f_{h}\left(\mathbf{p}_{i}\right), \quad x_{i}^{k}=f_{k}\left(\mathbf{p}_{i}\right), \quad y_{j}^{h}=f_{h}\left(\mathbf{n}_{j}\right), \quad y_{j}^{k}=f_{k}\left(\mathbf{n}_{j}\right) .
$$

The pAUCs for the two dichotomizers, considering the FPR interval $\left(0, t_{1}\right)$, are:

$$
p A U C_{h}=\frac{\sum_{i=1}^{m_{P}} \sum_{j=1}^{m_{N}} I\left(x_{i}^{h}>y_{j}^{h}, y_{j}^{h}>q_{y^{h}}^{t_{1}}\right)}{m_{P} m_{N}}, p A U C_{k}=\frac{\sum_{i=1}^{m_{P}} \sum_{j=1}^{m_{N}} I\left(x_{i}^{k}>y_{j}^{k}, y_{j}^{k}>q_{y^{k}}^{t_{1}}\right)}{m_{P} m_{N}}
$$

It is worth to note that finding the linear combination of two generic dichotomizers $f_{l c}=\alpha_{h} f_{h}+\alpha_{k} f_{k}$ such that maximizes the pAUC, is equivalent to find the weight $\alpha=\frac{\alpha_{k}}{\alpha_{h}} \in(-\infty,+\infty)$ which maximizes the pAUC for $f_{l c}=f_{h}+\alpha f_{k}$. Therefore, considering the linear combination, the outcomes on $\mathbf{p}_{i}$ and $\mathbf{n}_{j}$ are:

$$
\xi_{i}=f_{\mathrm{lc}}\left(\mathbf{p}_{i}\right)=x_{i}^{h}+\alpha x_{i}^{k}, \quad \eta_{j}=f_{\mathrm{lc}}\left(\mathbf{n}_{j}\right)=y_{j}^{h}+\alpha y_{j}^{k} .
$$

\footnotetext{
${ }^{1}$ The quantile function returns the value below which random draws from the negative population would fall, $\left(1-t_{k}\right) \times 100$ percent of the time.

${ }^{2}$ If $t_{0}=0$, then the $1-t_{0}$ quantile $q_{y}^{t_{0}}$ is equal to $+\infty$.
} 
and the pAUC is:

$$
p A U C_{\mathrm{lc}}=\frac{1}{m_{P} m_{N}}\left(\sum_{i=1}^{m_{P}} \sum_{j=1}^{m_{N}} I\left(\xi_{i}>\eta_{j},\left(\eta_{j}>q_{\eta}^{t_{1}}(\alpha)\right)\right)\right)
$$

In order to find the value $\alpha_{\text {opt }}$ which maximizes $p A U C_{\mathrm{lc}}$, let us analyze the term $I\left(\xi_{i}>\eta_{j}\right)$ without considering the constraint on the quantile. In particular, let us remind from [9] how it depends on the values of $I\left(x_{i}^{h}, y_{j}^{h}\right)$ and $I\left(x_{i}^{k}, y_{j}^{k}\right)$ :

- $I\left(x_{i}^{h}, y_{j}^{h}\right)=1$ and $I\left(x_{i}^{k}, y_{j}^{k}\right)=1$. In this case the two samples are correctly ranked by the two dichotomizers, and $I\left(\xi_{i}>\eta_{j}\right)=1$.

- $I\left(x_{i}^{h}, y_{j}^{h}\right)=0$ and $I\left(x_{i}^{k}, y_{j}^{k}\right)=0$. In this case neither dichotomizer ranks correctly the samples and thus the contribution for the $p A U C$ is 0 .

- $I\left(x_{i}^{h}, y_{j}^{h}\right)$ xor $I\left(x_{i}^{k}, y_{j}^{k}\right)=1$. Only one dichotomizer ranks correctly the samples while the other one is wrong. In this case the value of $I\left(\xi_{i}>\eta_{j}\right)$ depends on the weight $\alpha$.

The subset $\mathrm{T}$ can be divided into four subsets: $T_{h k}, T_{h \bar{k}}, T_{\bar{h} k}$ and $T_{\bar{h} \bar{k}}$ defined as:

$$
\begin{aligned}
& T_{h k}=\left\{\left(\mathbf{p}_{i}, \mathbf{n}_{j}\right) \mid I\left(x_{i}^{h}, y_{j}^{h}\right)=1 \text { and } I\left(x_{i}^{k}, y_{j}^{k}\right)=1\right\}, \\
& T_{\bar{h} k}=\left\{\left(\mathbf{p}_{i}, \mathbf{n}_{j}\right) \mid I\left(x_{i}^{h}, y_{j}^{h}\right)=0 \text { and } I\left(x_{i}^{k}, y_{j}^{k}\right)=1\right\}, \\
& T_{h \bar{k}}=\left\{\left(\mathbf{p}_{i}, \mathbf{n}_{j}\right) \mid I\left(x_{i}^{h}, y_{j}^{h}\right)=1 \text { and } I\left(x_{i}^{k}, y_{j}^{k}\right)=0\right\}, \\
& T_{\bar{h} \bar{k}}=\left\{\left(\mathbf{p}_{i}, \mathbf{n}_{j}\right) \mid I\left(x_{i}^{h}, y_{j}^{h}\right)=0 \text { and } I\left(x_{i}^{k}, y_{j}^{k}\right)=0\right\}
\end{aligned}
$$

Now, let us consider the constraint on the negative samples related to the quantile, and define the following set:

$$
\Gamma_{\alpha}=\left\{\left(\mathbf{p}_{i}, \mathbf{n}_{j}\right) \in P \times N \mid y_{j}^{h}+\alpha y_{j}^{k}>q_{\eta}^{t_{1}}\right\}
$$

where $q_{\eta}^{t_{1}}$ is the $1-t_{1}$ of $\eta$, which depends on the weight $\alpha$. If we define the sets $T_{h k}^{\prime}, T_{\bar{h} k}^{\prime}, T_{h \bar{k}}^{\prime}, T_{\bar{h} \bar{k}}^{\prime}$ as:

$$
\begin{array}{ll}
T_{h k}^{\prime}=T_{h k} \cap \Gamma_{\alpha}, & T_{\bar{h} k}^{\prime}=T_{\bar{h} k} \cap \Gamma_{\alpha}, \\
T_{h \bar{k}}^{\prime \prime}=T_{h \bar{k}} \cap \Gamma_{\alpha}, & T_{\bar{h} \bar{k}}^{\prime \prime}=T_{\bar{h} \bar{k}} \cap \Gamma_{\alpha},
\end{array}
$$

the expression for $p A U C_{\mathrm{lc}}$ in equation 8 can be written as:

$$
\begin{aligned}
& p A U C_{\mathrm{lc}}=\frac{1}{m_{P} m_{N}}\left(\sum_{\left(\mathbf{p}_{i}, \mathbf{n}_{j}\right) \in T_{\bar{h} \bar{k}}^{\prime}} I\left(\xi_{i}>\eta_{j}\right)+\sum_{\left(\mathbf{p}_{i}, \mathbf{n}_{j}\right) \in T_{h k}^{\prime}} I\left(\xi_{i}>\eta_{j}\right)\right. \\
& \left.+\sum_{\left(\mathbf{p}_{i}, \mathbf{n}_{j}\right) \in T_{h \bar{k}}^{\prime} \cup T_{\bar{h} k}^{\prime}} I\left(\xi_{i}>\eta_{j}\right)\right)=\frac{0+\gamma(\alpha)+\nu(\alpha)}{m_{P} m_{N}} .
\end{aligned}
$$

and the optimal weight is given by:

$$
\alpha_{\mathrm{opt}}=\arg \max _{\alpha}(\gamma(\alpha)+\nu(\alpha)) .
$$


In order to find $\alpha_{o p t}$, let us recall that $I\left(\xi_{i}>\eta_{j}\right)=1$ only if:

$$
\left(x_{i}^{h}-y_{j}^{h}\right)+\alpha\left(x_{i}^{k}-y_{j}^{k}\right)>0
$$

such that: $y_{j}^{h}+\alpha y_{j}^{k}>q_{\eta}^{t_{1}}(\alpha)$. If we define $\Delta_{i j}^{h}=x_{i}^{h}-y_{j}^{h}$ and $\Delta_{i j}^{k}=x_{i}^{k}-y_{j}^{k}$ and considering the three subsets $T_{h k}^{\prime}, T_{h \bar{k}}^{\prime}, T_{\bar{h} k}^{\prime}$, we obtain three different constraints:

$$
\alpha<-\frac{\Delta_{i j}^{h}}{\Delta_{i j}^{k}} \text { if }\left(\mathbf{p}_{i}, \mathbf{n}_{j}\right) \in T_{h \bar{k}}^{\prime}, \quad \alpha>-\frac{\Delta_{i j}^{h}}{\Delta_{i j}^{k}} \text { if }\left(\mathbf{p}_{i}, \mathbf{n}_{j}\right) \in T_{\bar{h} k}^{\prime}, \quad \alpha>-\frac{\Delta_{i j}^{h}}{\Delta_{i j}^{k}} \text { if }\left(\mathbf{p}_{i}, \mathbf{n}_{j}\right) \in T_{h k}^{\prime} .
$$

The pAUC is maximized when the number of pairs satisfying the previous constraints is maximized. Introducing the cumulative functions as follow:

$$
\begin{aligned}
& F_{h \bar{k}}^{\prime}(\alpha)=\operatorname{card}\left(\left(\mathbf{p}_{i}, \mathbf{n}_{j}\right) \in T_{h \bar{k}}^{\prime} \mid-\frac{\Delta_{i j}^{h}}{\Delta_{i j}^{k}}>\alpha\right) \\
& F_{\bar{h} k}^{\prime}(\alpha)=\operatorname{card}\left(\left(\mathbf{p}_{i}, \mathbf{n}_{j}\right) \in T_{\bar{h} k}^{\prime} \mid-\frac{\Delta_{i j}^{h}}{\Delta_{i j}^{k}}<\alpha\right) \\
& F_{h k}^{\prime}(\alpha)=\operatorname{card}\left(\left(\mathbf{p}_{i}, \mathbf{n}_{j}\right) \in T_{h k}^{\prime} \mid-\frac{\Delta_{i j}^{h}}{\Delta_{i j}^{k}}<\alpha\right)
\end{aligned}
$$

then, the function to be maximized is defined as:

$$
\gamma(\alpha)+\nu(\alpha)=F_{h \bar{k}}^{\prime}(\alpha)+F_{\bar{h} k}^{\prime}(\alpha)+F_{h k}^{\prime}(\alpha)
$$

It is worth to note, from the previous analysis, that $F_{h \bar{k}}^{\prime}$ and $F_{\bar{h} k}^{\prime}$ depend on the interaction between the pair values and $\alpha$, while $F_{h k}^{\prime}$ only depends on $\alpha$, due to the quantile values.

The optimal values can be easily found by means of linear search:

$$
\alpha_{\mathrm{opt}}=\arg \max _{\alpha}\left(F_{h \bar{k}}^{\prime}(\alpha)+F_{\bar{h} k}^{\prime}(\alpha)+F_{h k}^{\prime}(\alpha)\right)
$$

\section{Linear Combination of $K>2$ Dichotomizers}

The linear combination of $K>2$ dichotomizers is defined as:

$$
f_{l c}(x)=\alpha_{1} f_{1}(x)+\alpha_{2} f_{2}(x)+\ldots+\alpha_{K} f_{K}(x)=\sum_{i=1}^{K} \alpha_{i} f_{i}(x)
$$

In order to find the optimal weight vector $\alpha_{o p t}=\left(\alpha_{1}, \ldots, \alpha_{K}\right)$ that maximizes the pAUC associated to $f_{l c}(x)$, the method described in the previous section cannot be generalized in a computational feasible algorithm. Therefore, the proposed algorithm is based on the approximation of the solution by dividing the whole $K$-combination problem into a series of feasible pairwise combination problems using the greedy approach. 


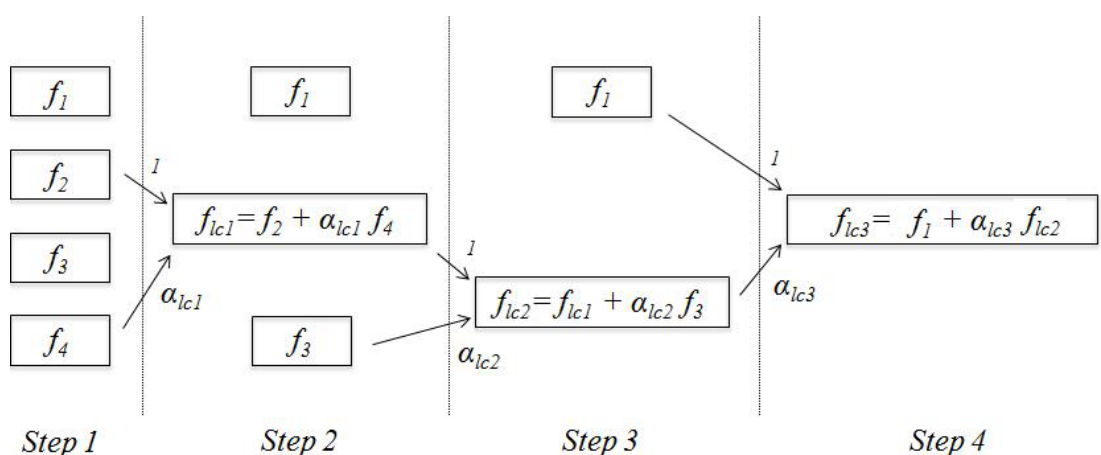

Fig. 1. Example of greedy approach steps

Table 1. XM2VTS database properties

\begin{tabular}{|l|r|r|r|}
\cline { 2 - 4 } \multicolumn{1}{c|}{} & \# Sample & \# Positive & \# Negative \\
\hline Validation Set & 40600 & 600 & 40000 \\
\hline Test Set & 112200 & 400 & 111800 \\
\hline
\end{tabular}

Table 2. pAUC of each classifier, calculated in step 1 and step 2 in fig. 1

(a) Step 1

\begin{tabular}{|c|c|c|c|c|}
\cline { 2 - 5 } \multicolumn{1}{c|}{} & $f_{1}$ & $f_{2}$ & $f_{3}$ & $f_{4}$ \\
\hline pAUC & 0.093 & 0.095 & 0.094 & 0.096 \\
\hline
\end{tabular}

(b) Step 2

\begin{tabular}{|c|c|c|c|}
\cline { 2 - 4 } \multicolumn{1}{c|}{} & $f_{l c 1}$ & $f_{1}$ & $f_{3}$ \\
\hline pAUC & 0.097 & 0.093 & 0.094 \\
\hline
\end{tabular}

The greedy approach is a suboptimal method which uses $K-1$ steps of a simpler algorithm, obtaining the optimum weights only after the $K-1$ steps. In particular, in each step two dichotomizers are combined using the algorithm described in section 3 finding the optimal weight for that combination. After the first weight is computed, the number of dichotomizers decreases from $K$ to $K-1$. After that, there is the choice of the two dichotomizers for the next step. This procedure is repeated until all the dichotomizers have been combined.

Using the greedy approach is equivalent to find a suboptimal solution by making a locally optimal choice. Therefore, in each iteration, the choice of two dichotomizers that should be combined, plays an important role. In fact it is a fundamental issue that can affect the performance of the algorithm.

In the proposed method, we consider an approach based on the best performance of the individual dichotomizer in terms of pAUC. Therefore, at each step, the algorithm chooses the two dichotomizers with the maximum pAUC values.

Figure 1 and table 2 show the steps of the algorithm considering the interval $F P R=(0,0.1)$, and assuming the linear combination of 4 classifiers. First of all, for each classifier the pAUC value is computed (tab. 2(a)). Then, the pair that corresponds to the two classifiers with higher pAUCs, in the example $f_{2}$ and $f_{4}$, 
is used to find the $\alpha_{l c 1}$ for the first linear combination. The vector of pAUCs is updated (tab. 2(b)), considering the new classifier $f_{l c 1}$. Then, these steps are repeated until there are no more classifiers to be combined.

In order to recover the weight for each of the classifiers, a combination tree is built during the evaluation of the $\alpha_{\text {opt }}$ (fig. 1). The original classifiers constitute the leaves of the tree and, each time a pair of classifiers is combined, a parent node is added associated to the two combined classifiers. The edges are labeled with the weights assigned to each classifier. At the end of the computation, the weight of each classifier can be easily recovered by traversing the tree from the leaf up to the root and multiplying all the values found on the edges. In the example shown in figure 1 the final combination of classifiers is:

$$
\begin{gathered}
f_{l c 3}=f_{1}+\alpha_{l c 3} f_{l c 2}=f_{1}+\alpha_{l c 3}\left(f_{l c 1}+\alpha_{l c 2} f_{3}\right) \\
=f_{1}+\alpha_{l c 3}\left(f_{2}+\alpha_{l c 1} f_{4}+\alpha_{l c 2} f_{3}\right)=f_{1}+\alpha_{l c 3} f_{2}+\alpha_{l c 3} \alpha_{l c 1} f_{4}+\alpha_{l c 3} \alpha_{l c 2} f_{3}
\end{gathered}
$$

and the final weight vector is given by: $\alpha_{o p t}=\left(\begin{array}{lllll}1 & \alpha_{l c 3} & \alpha_{l c 2} \alpha_{l c 3} & \alpha_{l c 1} \alpha_{l c 3}\end{array}\right)$.

\section{$5 \quad$ Experimental Results}

In order to evaluate the performance of the pROC algorithm proposed, the experiments are performed on the public-domain biometric dataset XM2VTS [10], characterized by 8 matchers, using the partition of the scores into training and test set proposed in [10] and showed in table 1]. The XM2VTS is a multimodal database containing video sequences and speech data of 295 subjects recorded in four sessions in a period of 1 month. In order to assess its performance the Lausanne protocol has been used to randomly divide all the subjects into positive and negative classes: 200 positive, 25 evaluation negatives and 70 test negatives. All the details about the procedure used to obtain the final dichotomizers are described in [10].

The combination rule proposed (pROC) is compared with other algorithms: a method proposed by $\mathrm{Su}$ and $\mathrm{Liu}$ in [11, that provides a linear combination to maximize the AUC assuming a normal distribution for both positive and negative samples, and the DROC method proposed in [9] that maximizes the AUC considering the pairs of positive and negative samples that contribute to the AUC values without any assumption on their distribution. It is worth to note that both of the algorithms considered for the comparison evaluate a weight vector such that the combination maximizes the AUC. Furthermore, another considered combination rule is the average of the outcomes of the dichotomizers which is independent from any direct maximization of the metric and from any distribution.

For each considered method the vector $\alpha$ of coefficients for the linear combination is evaluated on the validation set, and then applied to the test set. The results are analyzed in term of partial AUC, considering the false positive ranges: $F P R_{0.1}=(0,0.1), F P R_{0.05}=(0,0.05)$ and $F P R_{0.01}=(0,0.01)$. 


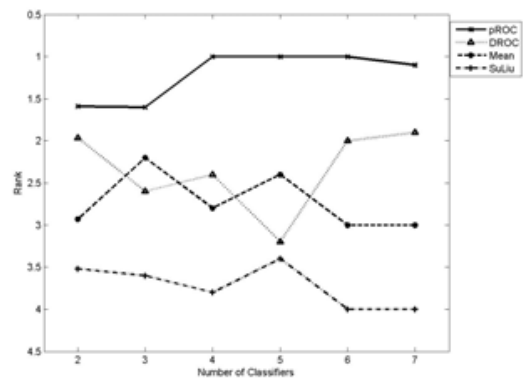

(a)

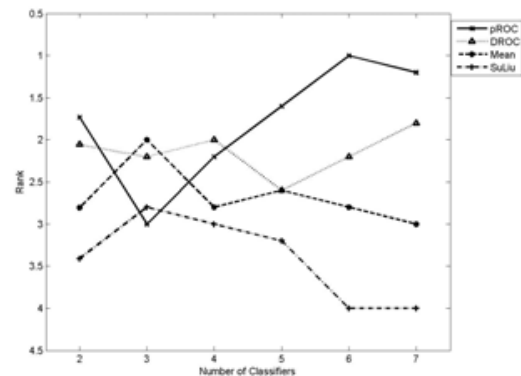

(b)

Fig. 2. Mean of rank on validation set (a) and test set (b), with $F P R_{0.1}$. Note that in both figures the $\mathrm{y}$-axis is reversed.

The number of combined dichotomizers varies from 2 to 7 . For each of those experiments we obtain different number of possible combinations that are independent from each other. Therefore, we use an approach based on giving a rank to each method compared to the others, for each independent experiment. Let us consider the pAUC values $\left\{p A U C_{i j}\right\}_{M \times L}$, for $i=1, \ldots, M$ with $M$ the number of combinations, and for $j=1, \ldots, L$ with $L$ number of combination rules that are compared. For each row we assign a rank value $r_{j}^{i}$ from 1 to $L$ to each column depending on the pAUC values: the highest pAUC gets rank 1, the second highest the rank 2 , and so on until $L$ (in our case $L=4$ ). If there are tied pAUCs, the average of the ranks involved is assigned to all pAUCs tied for a given rank. Only in this case it is appropriate to average the obtained ranks on the number of combinations:

$$
\bar{r}_{j}=\frac{1}{M} \sum_{i=1}^{M} r_{j}^{i}
$$

Figures 2(a)-4(a) and figures 2(b)-4(b) show the results on the validation set and test set, respectively, varying the FPR ranges. The higher the curve, i.e. the lower the value, the better the related method.

Analyzing the results, we can observe a very good generalization of the algorithm pROC except for $F P R_{0.1}$, where its behavior is comparable with the one of the other methods. Notwithstanding that, the algorithm performs well on the most part of the experiments. Decreasing the FPR range, pROC performance are much better since the algorithm is more adapt to the problem.

Moreover, it is shown the difference between the two kinds of maximization: AUC-based and pAUC-based. In particular, it is worth to note that methods designed to maximize the AUC (DROC and SuLiu) do not maximize the pAUC. In fact, DROC and SuLiu maximize the performance measure considering all the range of FPR, while pROC considers only a particular range of it.

Furthermore, assuming a normal distribution of negative and positive samples in SuLiu, does not perform as good as the average rule, and as DROC 


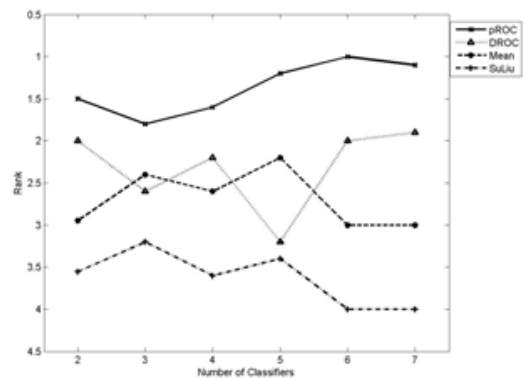

(a)

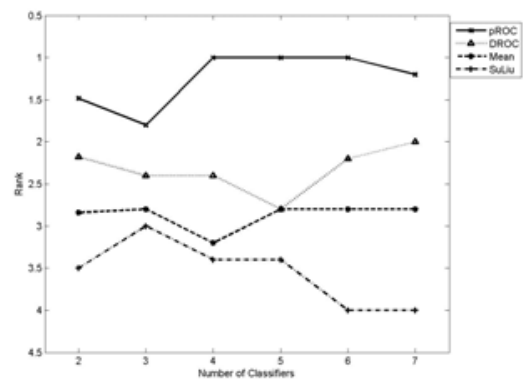

(b)

Fig. 3. Mean of rank on validation set (a) and test set (b), with $F P R_{0.05}$

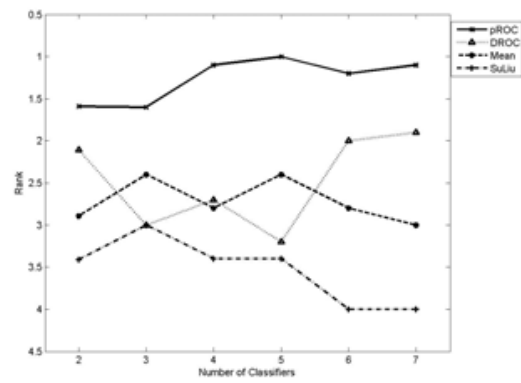

(a)

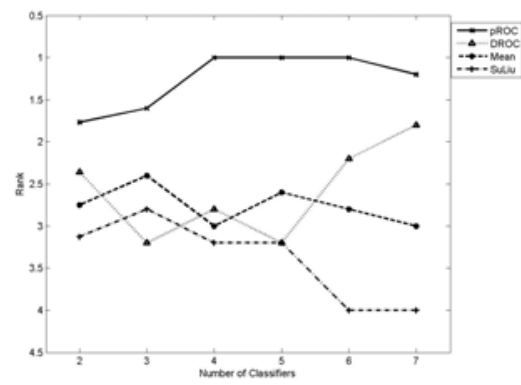

(b)

Fig. 4. Mean of rank on validation set (a) and test set (b), with $F P R_{0.01}$

method which is independent from any distributions. Such difference is due to the fact that the computation of the weight is affected more when the assumed distribution model is not close to the real one. In addition to the fact that the AUC-based method SuLiu does not maximize the pAUC, it has also less performance than a method that is independent from any kind of maximization (the average method).

\section{Conclusions}

In this paper, we have proposed a new linear combination method aims to improve the partial Area under the ROC curve (pAUC) in a two-class classification problem, since little attention has been given to the use of pAUC in machine learning and specifically as a performance measure in combining classifiers.

The algorithm designed to maximize the pAUC is based on the dependence of the metric on the coefficients vector $\alpha$ used for the linear combination of dichotomizers. The algorithm has been implemented for a two dichotomizers combination, then extended to the combination of $K>2$ dichotomizers. 
The results obtained have shown good performance of pROC method compared with other algorithms. In particular, it has been noticed that maximizing the total AUC is not so effective for the maximization of the partial AUC, in fact maximizing the metric on all the range of $\mathrm{FPR}$ is not equivalent to maximize the metric in a portion on that range. Moreover, methods that assume a particular distribution model for negative and positive samples, are not able to perform as good as method that do not have any assumption.

Future work regards an analysis on the possible rules that can be used in the greedy approach in order to choose the dichotomizers to combine at each step. It will be interesting to note if the performance will change and how.

\section{References}

1. Huang, J., Ling, C.X.: Using AUC and accuracy in evaluating learning algorithms. IEEE Trans. on Knowledge and Data Engineering 17, 299-310 (2005)

2. Cortes, C., Mohri, M.: AUC optimization vs. error rate minimization advances. In: Neural Information Processing Systems. MIT Press, Cambridge (2003)

3. Dodd, L.E., Pepe, M.S.: Partial AUC estimation and regression. Biometrics 59, 614-623 (2003)

4. Jiang, Y., Metz, C.E., Nishikawa, R.M.: A receiver operating characteristic partial area index for highly sensitive diagnostic tests. Radiology 201, 745-750 (1996)

5. McClish, D.K.: Analyzing a portion of the ROC curve. Medical Decision Making 9, 190-195 (1989)

6. Bradley, A.P.: The use of the area under the roc curve in the evaluation of machine learning algorithms. Patt. Recogn. 30, 1145-1159 (1997)

7. Hanley, J.A., McNeil, B.J.: The meaning and use of the area under a receiver operating characteristic (ROC) curve. Radiology 143, 29-36 (1982)

8. Nandakumar, K., Dass, S.C., Jain, A.K.: Likelihood ratio-based biometric score fusion. IEEE Trans. on Patt. Anal. and Mach. Intell. 30, 342-347 (2008)

9. Marrocco, C., Molinara, M., Tortorella, F.: Optimal linear combination of dichotomizers via auc. In: Proceed. of the 22nd Intern. Conf. on Mach. Learn. Workshop on ROC Analysis in Mach. Learn., pp. 778-785 (2005)

10. Poh, N., Bengio, S.: Database, protocol and tools for evaluating score-level fusion algorithms in biometric authentication. Patt. Recogn. 39, 223-233 (2006)

11. Su, J.Q., Liu, J.S.: Linear combinations of multiple diagnostic markers. Journal of the Americ. Stat. Assoc. 88, 1350-1355 (1993) 